\title{
Multi-criteria Optimization of the Mechanical Systems by Virtual Prototyping Tools
}

\author{
Cătălin Alexandru
}

\begin{abstract}
The work approaches the multi-criteria optimization with design constraints of the mechanical systems by using virtual prototyping software environment. The optimization problem is formulated in the following way: to find the optimal values of the independent design variables, defined by upper and lower bounds, with the view to minimize or maximize the design cost function (i.e. design objective), which is subject to equations of state for the mechanical systems, and design constraints. The application is developed for the hood folding mechanism of a storage container, the optimization process being configured by one design objective, and three design constraints that cover the whole range motion of the folding mechanism. The virtual prototyping environment ADAMS of MSC Software has been used for the modeling, simulation, and optimization of the mechanical system.
\end{abstract}

Index Terms-Mechanical system, optimization, design objective, design constraint, virtual prototyping.

\section{INTRODUCTION}

The development of automatic analysis \& optimization tools is a continuous concern and challenge for the research in the field of mechanical systems design. Important publications reveal a growing interest in analysis \& optimization methods for multi-body systems (MBS), which may facilitate the self-formulating algorithms [1]-[6]. Such methods are intensively used to develop powerful virtual prototyping software environments.

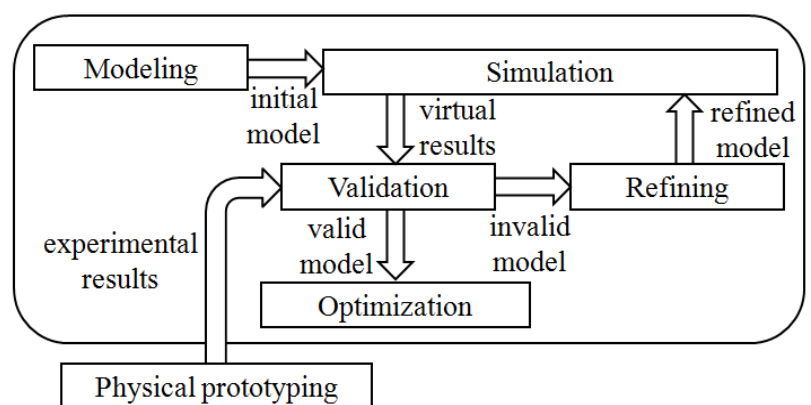

Fig. 1. The virtual prototyping platform.

A typical virtual prototyping platform (process) is schematically represented in figure 1 . The stages to create a virtual prototype mirror the stages to create a physical model [7], [8], as follows: modeling (create parts, restrictions, and forces generating elements), simulation (analysis / testing), validation (compare virtual and experimental data), refining

Manuscript received March 3, 2017; revised April 17, 2017.

C. Alexandru is with the Product Design, Mechatronics and Environment Department, Transilvania University of Braşov, 500036 Braşov, Romania (e-mail: calex@unitbv.ro). (improve the fidelity of the virtual model in relation to the physical one, e.g. add friction, flexible parts, actuating \& control systems [9]), optimization (improving the behavior of the mechanical system, usually from - but not limited to functional point of view). The physical (hardware) prototyping is a support activity for the virtual (software) prototyping, providing the experimental results used in the validation stage of the virtual model [10]. The basic principle for a successful virtual prototyping process is to manipulate the simplifying assumptions in a way that reduces the complexity of the virtual model, but without altering the accuracy of the results [11].

In these terms, the current work is focused on the optimization stage of the mechanical systems in the virtual prototyping concept. First of all, in the $2^{\text {nd }}$ section of the paper, the optimization algorithm is depicted in the main specific steps, and then, in the $3^{\text {rd }}$ section, the algorithm is implemented for the optimal design of a complex mechanical system (namely, the hood folding mechanism of a storage container), whose behavior is subject of several functional constraints. Results and conclusions are discussed formulated in the $4^{\text {th }}$ section, with the aim to prove the viability (usefulness) of the optimization study.

The optimization purpose is to minimize the material cost of the mechanical system while satisfying three design constraints that refer to specific functional requirements throughout the entire range of motion of the mechanism. The virtual prototyping environment ADAMS (Automatic Dynamic Analysis of Mechanical Systems) of MSC Software has been used for performing the optimization.

\section{The OPtimiZATION ALGORITHM}

In the virtual prototyping concept, the optimization of the mechanical systems is performed with the following steps [12]: parameterizing the virtual model, defining the design variables, defining the design objectives and constraints for optimization, performing parametric studies or design of experiments, and optimizing the model on the basis of the main design variables.

Usually, the parameterization of the mechanical systems is made by using the points that define the structural model, in fact the locations of the geometric constraints (i.e. the joints). The parameterization simplifies changes to model because it helps to automatically resize, relocate and orient parts. In this way, relationships into the model are created, so that when a point is changed, any other objects (bodies, joints, forces) that depend on it will be updated.

Design variables represent elements in model that allow creating independent parameters and tie modeling objects to 
them. Usually, the locations (coordinates) of the design points are considered as design variables. Design variable allows running automated simulations that vary the values of the variable over specified ranges to understand the sensitivity of the design objective to the design variable changes, or to find the optimal values.

In addition, using design variables, parametric studies can be performed. The parametric study represents a set of simulations that help to adjust a parameter to measure its effect on the performance of the mechanical system. Design study describes the ability to select a design variable, sweep the variable through a range of values, and then simulate the behavior of the various designs in order to understand the sensitivity of the overall system to these design variations. As result, design study allows to identify the main design variables, with great influence on the mechanical system behavior.

In general, an optimization problem is described as a problem to minimize or maximize an objective function over a selection of design variables, while satisfying various constraints on the design and state variables of the system. Various algorithms are available for finding a solution to an optimization problem, given the problem has been formulated in the manner described in this section.

The objective function is a numerical representation of the quality, efficiency, cost, or stability of the mechanical system. The design engineer decides whether the optimization chooses to find the minimum or maximum of the function. The optimal value of this function corresponds to the best possible design in the given conditions. Examples of objective functions include execution time, energy (effort) required, and total material costs.

The objective function, also called the cost functional, performance measure, or performance index, is a numerical quantification that distinguishes or rates candidate designs. The optimal design is achieved when the objective function is minimized or maximized. In the case of minimization, the objective grows smaller as the design improves. In the case of maximization, the objective grows larger as the design improves. Typical standard objective functions may refer to minimum operating time, minimum effort or energy consumption, maximum energetic or economic efficiency, and others.

Design variables can be thought of as the unknowns for the design problem. These are the parameters that can alter to define the design. Changes in the design variables should result in changes to the design function. Examples of design variables are part dimensions, joints locations, part masses and others.

To avoid unacceptable results/configurations, from functional or constructive point of view, design constraints for optimization can be defined. The optimization study improves the design objective as much as possible without violating the design constraints. Constraints are boundaries that, directly or indirectly, eliminate unacceptable designs. Constraints often take the form of additional goals for the optimization of the mechanical system.

Usually, a constraint creates an inequality relation, the optimization keeping the value of the constraint less than or equal to zero. Constraints can involve the simulation results, but are not required to do so. They can constrain overall size, weight, or other factors that depend only on model data. Most designs have specific requirements, such as to support a specified load or trace a specified path. There is usually some means of distinguishing a good design from a bad design: it is too heavy or it will cost too much to produce. Some requirements can be restrictions on a design: packaging, end conditions, or material availability.

Part of the design process is to manipulate the unknowns (variables) in a design to arrive at a good design that satisfies all goals (objectives) and restrictions (constraints). If these features can be quantified, optimization techniques can be used to analytically arrive at the best theoretical solution.

In the general case, an optimization problem can be briefly formulated in the following way:

- to find the design variables: $a^{t}=\left[a_{1}, a_{2}, a_{3}, \ldots . a_{n}\right]^{t}$

- with the upper and lower bounds

$\Psi_{2+\mathrm{i}}=\mathrm{a}_{\mathrm{i}}-\left(\mathrm{a}_{\mathrm{i}}\right)^{\mathrm{H}} \leq 0, \mathrm{i}=1, \ldots, \mathrm{n}$

$\Psi_{3+\mathrm{i}}=\left(\mathrm{a}_{\mathrm{i}}\right)^{\mathrm{L}}-\mathrm{a}_{\mathrm{i}} \leq 0, \mathrm{i}=1, \ldots, \mathrm{n}$

where $\left(a_{i}\right)^{\mathrm{H}}$ is upper bound on the $i$-th design variable, and $\left(a_{i}\right)^{L}$ - lower bound on the $i$-th design variable

- to minimize/maximize design cost function:

$\Psi_{0}=\mathrm{f}(\mathrm{y}, \mathrm{a}, \mathrm{t})$

- subject to

a. equations of state for the mechanical systems $\mathrm{g}(\mathrm{y}, \mathrm{a}, \mathrm{t})=0$

b. design constraints

b.1. equality constraints

$\Psi \mathrm{c}=\mathrm{h}_{\mathrm{c}}(\mathrm{y}, \mathrm{a}, \mathrm{t})=0$

b.2. inequality constraints

$\Psi \mathrm{i}=\mathrm{h}_{\mathrm{i}}(\mathrm{y}, \mathrm{a}, \mathrm{t}) \leq 0$

where: $\mathrm{t}$ - time, $\mathrm{y}$ - state vector for the system, $\mathrm{g}$ - vector of governing equations, $h_{c}$ - vector of design functions defining equality constraints, $h_{i}$ - vector of design functions defining inequality constraints [13].

For this paper, the optimization study will be performed by using the optimal design tools provided by MSC.ADAMS, so that there is no need to develop a mathematical algorithm for the numerical optimization.

\section{CASE STUdY}

For this paper, the hood folding mechanism for a storage container has been considered. The virtual model of the mechanism, which is shown in Fig. 2, has been developed by using the MBS package ADAMS of MSC Software [14].

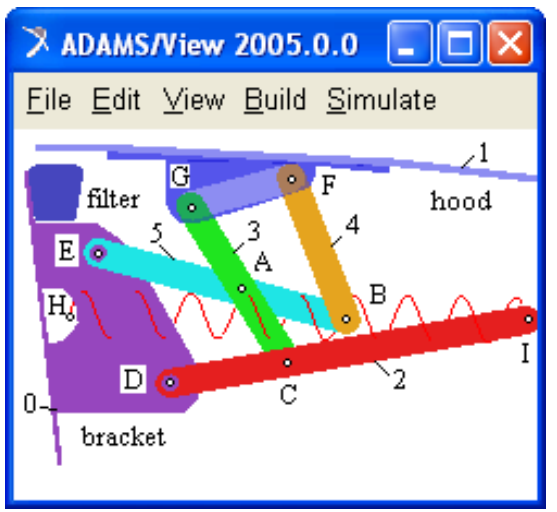

Fig. 2. The MBS virtual model of the hood folding mechanism. 
The mechanism contains five mobile bodies (1-5), the fixed part (0) of the model being the container bracket. The six bodies are connected through revolute joints (A-G). In addition, the mechanism includes a spring, which is connected (in $\mathrm{H}$ and I) between the $2^{\text {nd }}$ link and the bracket. The storage compartment has a filter that needs to be replaced, therefore the mechanism must be designed so that the hood does not interfere with the filter when it is opened.

In the virtual model, there are nine design points that control the locations of the joints (A - 3/5, B - 4/5, C - 2/3, D $2 / 0, \mathrm{E}-5 / 0, \mathrm{~F}-1 / 4$, and $\mathrm{G}-1 / 3$ ) and the locations in which the spring is connected to the adjacent bodies ( $\mathrm{H}$, and $\mathrm{I}$ ). Considering that the locations of the points on bracket, which is attached to ground, and hood are established on constructive criteria, only four design points will be available for optimization: A, B, C, and I.

These points determine in fact four design variables: the lengths and the position angles (relative to the horizontal axis) of the links 2 and $5\left(\mathrm{~L}_{2}, \mathrm{~L}_{5}, \alpha_{2}, \alpha_{5}\right)$. The design variables allow to define independent parameters that can be linked to objects, and to organize the critical parameters of the design into a concise list of values that can be easily modified. In this way, a parametric model of the mechanism has been created.

The goal of the optimization is to minimize the material cost of the hood folding mechanism while satisfying the following three design constraints:

1) The hood does not interfere with the filter throughout the entire range of motion;

2) The inclination angle is of $50^{\circ}$ when the hood is fully open;

3) The filter is accessible from above when the hood is open.

The first design constraint is to design the linkage so that the hood never touches the filter as it is opened. The design function is based on a measure that defines the vertical distance from a marker on the rear of the hood - MARKER_I to the top surface of the filter - MARKER_J (fig. 3). In ADAMS, this measure was created by using in Function Builder the predefined function "Distance along $\mathrm{Y}$ ". When the value of the measure becomes negative, the hood penetrates the filter and this is an unacceptable design.

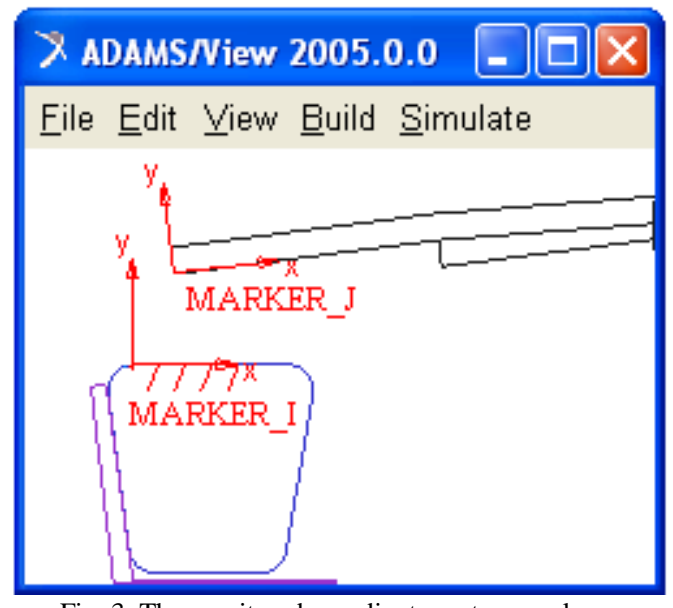

Fig. 3. The monitored coordinate system markers.

In ADAMS optimization objectives and constraints, the sign of a measure (+ or -) has special meaning [14]. For example, if an optimization constraint is positive, then it has been violated. If it is negative, it has not been violated, and the design is acceptable. In these terms, it is necessary to negate the DY function expression so that a positive value of the measure indicates that the hood marker has interfered with the filter's top surface. Conversely, if the measure stays negative throughout the operation cycle, it means that there is an acceptable design. Thus, the measure has the expression: distance_Y = -DY(Marker_I, Marker_J), the constraint applying to the maximum value of the measure.

The second design constraint is to have the hood open to a minimum inclination angle of $50^{\circ}$, so it is necessary to create a design constraint in order to monitor the angle, making sure it is satisfied during the optimization. The current inclination angle of the hood relative to ground is created by using in Function Builder the predefined function "Angle about Z" and then subtract it from the desired angle. The measure was created by referencing the marker placed in the front part of the hood (Fig. 4).

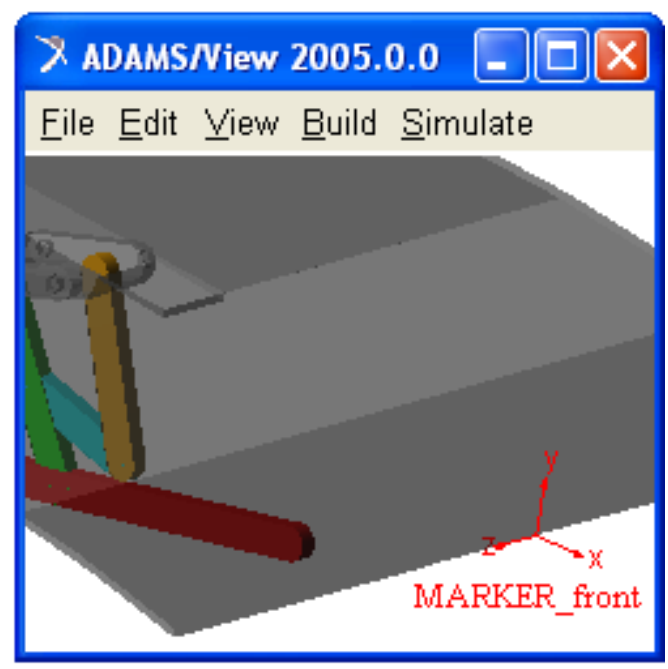

Fig. 4. The monitored marker in the front part of the hood.

Thus, the measure expression is: inclination_angle = 50-AZ(marker_front). This will result in a positive value for the measure when the angle is less than $50^{\circ}$. The second design constraint was created using the value at simulation end of this measure.

The third optimization constraint is to design the linkage such that the air filter is easily accessible from above when the hood is fully open. One way to achieve this goal is to have the rearward end of the hood move forward as the hood opens, ending at $50 \mathrm{~mm}$ offset from the starting configuration. The current horizontal distance between the coordinate system markers I and J (see Fig. 3) was created using the "Distance along X" function, and then it was subtracted from the desired offset distance of $50 \mathrm{~mm}$. In this way, the measure has the expression: distance_X $=50-\mathrm{DX}($ Marker_I, Marker_J). This result is a positive value for the measure when the distance is less than $50 \mathrm{~mm}$.

The above-created measure is used to define the third design constraint for the optimization process. When the constraint value is negative, the criterion is satisfied.

The goal of the optimization is to minimize the material cost. The cost of the material is proportional to the overall mass of the link parts. There was created a measure that sums up the total mass of the linkage using a computed measure. 


\section{RESUlTS \& CONCLUSIONS}

For the initial mechanism, defined by the initial values of the design variables: $\mathrm{L}_{2}=180 \mathrm{~mm}, \mathrm{~L}_{5}=230 \mathrm{~mm}, \alpha_{2}=-15^{\circ}$, and $\alpha_{5}=4.8^{\circ}$, the specific results are shown in figure 5 . There are the following approximate values of the design objective \& constraints:

- design objective $\rightarrow 1.141 \mathrm{~kg}$,

- $1^{\text {st }}$ constraint $\rightarrow 9.853 \mathrm{~mm}$,

- $2^{\text {nd }}$ constraint $\rightarrow 9.746^{\circ}$,

- $3^{\text {rd }}$ constraint $\rightarrow 28.198 \mathrm{~mm}$.

The results show that in the initial configuration the hood folding mechanism does not respect the imposed design constraints, all the three constraints being violated.
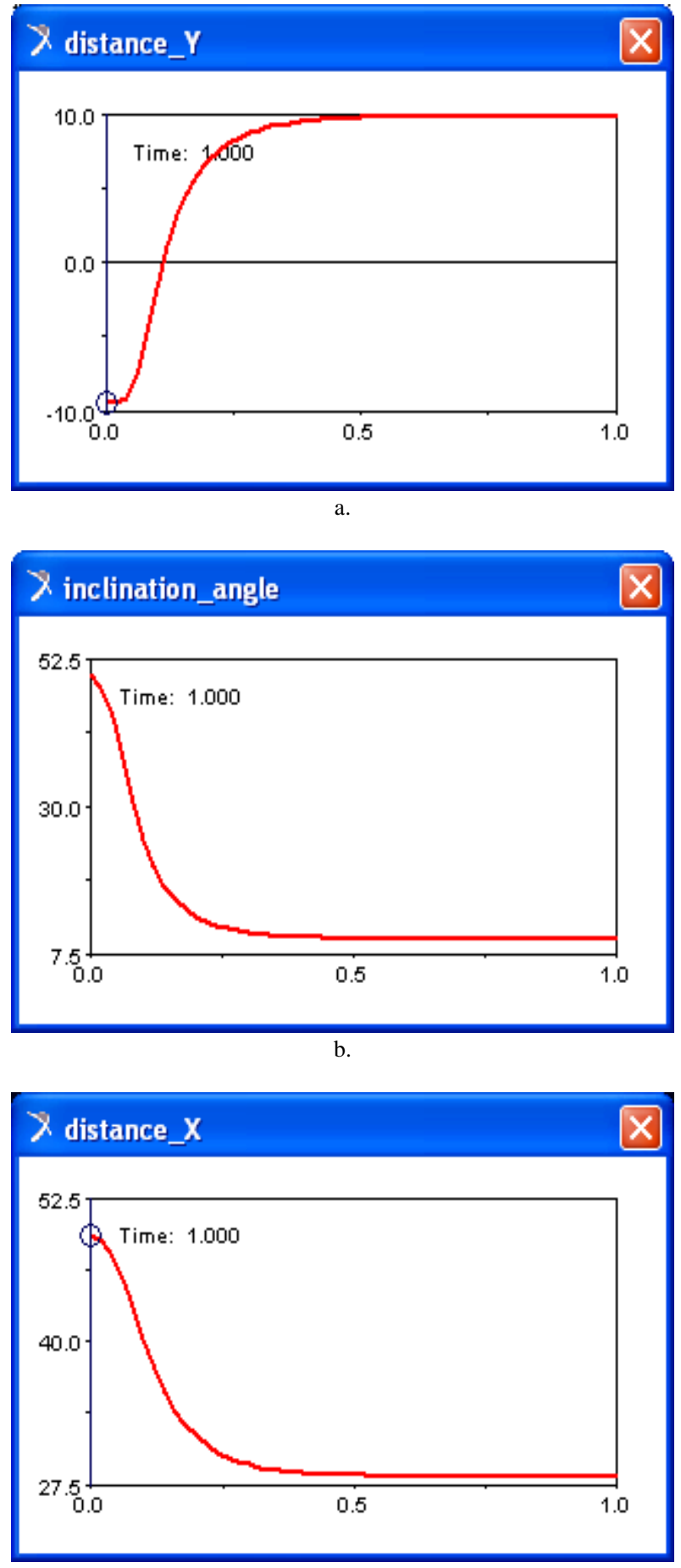

c.

Fig. 5. The variations of the constraint measures in the initial design.
The algorithm used to perform the optimization is OPTDES-GRG (Generalized Reduced Gradient), which is provided with ADAMS. This algorithm requires that design variables have range limits, since it works in scaled space. The differencing increment, which specifies the size of increment to use when performing finite differencing to compute gradients, is defined using the forward method (the value is added to the nominal value of each design variable on successive runs) [14].

For the optimized mechanism, the specific results are presented in figure 6 , with the following numerical values:

1) Design objective $\rightarrow 1.126 \mathrm{~kg}$,

2) $1 \mathrm{st}$ constraint $\rightarrow-5.945 \mathrm{E}-002 \mathrm{~mm}$,

3) 2nd constraint $\rightarrow-3.947 \mathrm{E}-003^{\circ}$,

4) 3rd constraint $\rightarrow-9.578 \mathrm{E}-002 \mathrm{~mm}$.

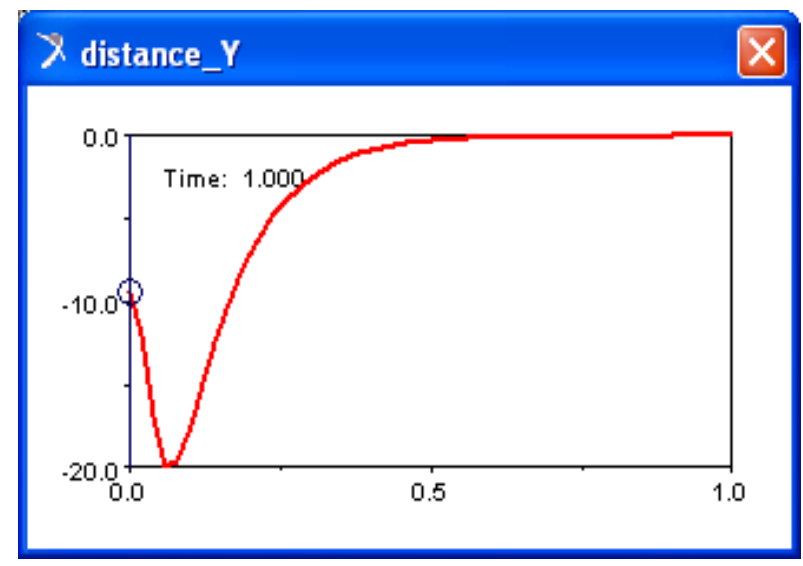

a.
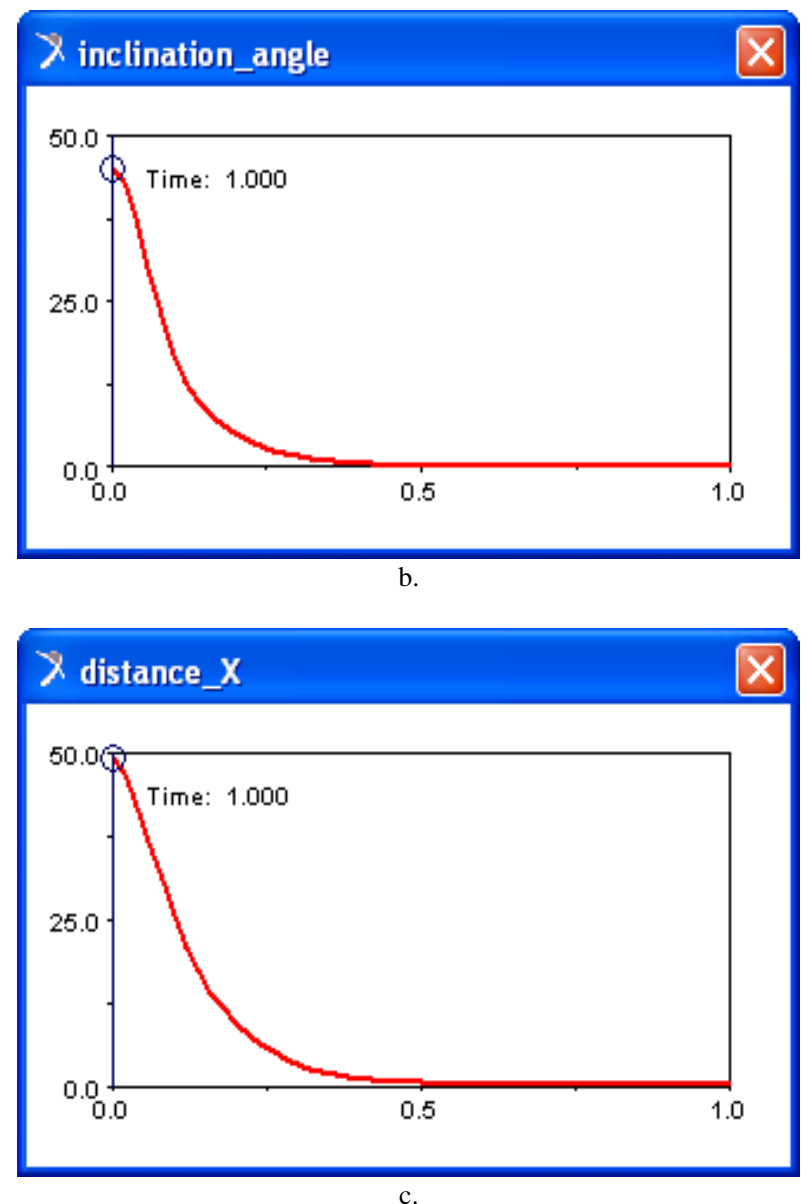

Fig. 6. The variations of the constraint measures in the optimal design. 
The optimal configuration of the mechanism is defined by the following values of the design variables: $\mathrm{L}_{2}=178.22 \mathrm{~mm}, \mathrm{~L}_{5}$ $=230 \mathrm{~mm}, \alpha_{2}=-14.97^{\circ}, \alpha_{5}=10^{\circ}$.

From the results shown in figure 6 , there can be observed that the optimized mechanism respects the design constraints, and at the same time there is a decrease in the material cost / mass. Therefore, the optimization objective is allowed, respecting the design constraints.

Finally, in figure 7 there are shown the graphic animation frames in the fully-open configuration of the initial (a) and optimized (b) mechanism.

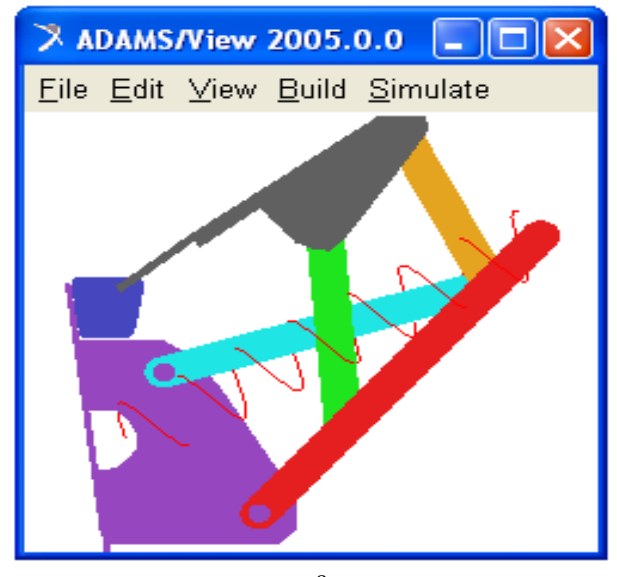

a.

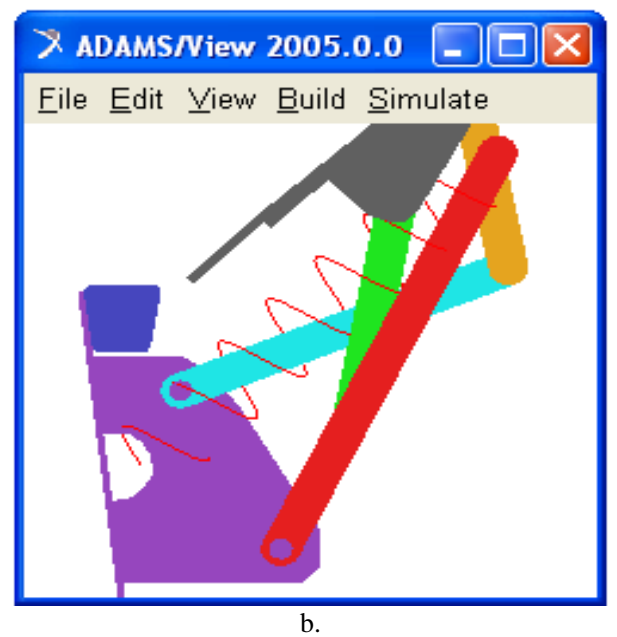

Fig. 7. The fully-open configuration of the mechanism.

In this work, specific aspects regarding the optimization of the mechanical systems by using virtual prototyping-based software tools have been presented. An important advantage of this kind of approach consists in the possibility of performing easy virtual measurements at any point and/or area of the mechanical system, and for any parameter. This is not always possible in the real cases due to the lack of space for sensors placement, lack of appropriate sensors, or high temperature in the fitting area of the sensor.

The optimization of the hood folding mechanism, which leads to a significantly improved mechanism, with minor changes in its geometric configuration, proves the usefulness of the adopted optimal design strategy. The behavioral performance predictions are obtained much earlier in the design process of the mechanical systems, thus allowing more effective and cost efficient design changes.

\section{REFERENCES}

[1] C. Alexandru, "Software platform for analyzing and optimizing the mechanical systems," Proceedings of the 10th IFToMM International Symposium on Science of Mechanisms and Machines - SYROM, pp. 665-677, Braşov, 2009.

[2] E. Eich-Soellner and C. Führer, Numerical Methods in Multibody Dynamics, Teubner, 2008.

[3] E.J. Haug and K.K. Choi, "Virtual prototyping simulation for design of mechanical systems," Transaction of ASME, no. 117, pp. 63-70, 1995.

[4] N.V. Orlandea, "ADAMS - theory and applications," Vehicle System Dynamics, no. 16, pp. 121-166, 1987.

[5] A.A. Shabana, Dynamics of Multibody Systems - Second Edition, John Wiley \& Sons, 1998.

[6] Ş. Staicu, "Dynamics of the 6-6 Stewart parallel manipulator," Robotics and Computer-Integrated Manufacturing, vol. 27, no. 1, pp. 212-220, 2011.

[7] D. Tarniţă and D. Marghitu, "Analysis of a hand arm system," Robotics and Computer-Integrated Manufacturing, vol. 29, pp. 493-501, 2013.

[8] D. Tarniță, M. Catană, and D.N. Tarniță, "Contributions on the modeling and simulation of the human knee joint with applications to the robotic structures", Mechanisms and Machine Science, vol. 20, pp. 283-297, 2014.V.N.

[9] T. Mikolajczyk, A. Olaru, and P. Krainski, "Adaptive control system for drill machine," Applied Mechanics and Materials, vol. 436, pp. 445-450, 2013.

[10] C. Berceanu, D. Tarniţă, and D. Filip, "About an experimental approach used to determine the kinematics of the human finger," Journal of the Solid State Phenomena, Robotics and Automation Systems, vol. 166-167, pp. 45-50, 2010.

[11] I. Barbu, "Virtual prototyping tools applied in mechanical engineering," Mecatronica, vol. 3, pp. 9-12, 2004.

[12] C. Alexandru, "Optimal design of the mechanical systems using parametric technique \& MBS (Multi-Body Systems) software," Advanced Materials Research, vol. 463-464, pp. 1129-1132, 2012.

[13] Sohoni and E. J. Haug, "A state space technique for optimal design of mechanisms," ASME Journal of Mechanical Design, vol. 104, no. 4, 1982.

[14] *** Getting Started Using ADAMS/View, MSC Software, 2005.

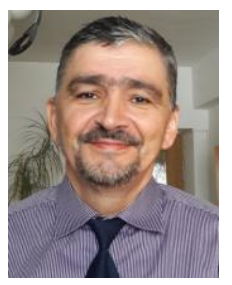

Cătălin Alexandru was born in Cetățeni, Romania, 1967. He received his bachelor in 1991, PhD (1998), $\mathrm{PhD}$ supervisor (2009) in mechanical engineering at Transilvania University of Braşov, Romania.

He is currently professor at Transilvania University of Braşov, Romania, in the Product Design, Mechatronics and Environment Department. $\mathrm{He}$ published over 150 scientific articles, part of them in prestigious ISI-rated journals (such as Mechanism and Machine Theory, Applied Mathematical Modelling, Journal of Renewable and Sustainable Energy, The Scientific World Journal, Proceedings of the Institution of Mechanical Engineers - Part C: Journal of Mechanical Engineering Science). He owns 2 patents. He participated at numerous international conferences in Romania and abroad (England, Scotland, Portugal, Spain, France, Germany, Austria, Italy, and others). Research interests in mechanical \& mechatronic systems, automotive engineering, renewable energy systems, virtual prototyping.

Prof. Alexandru is a member of International University Association for Science and Technology of Romania (UASTRO), and International Federation for the Promotion of Mechanism and Machine Science (IFToMM), editor for the Bulletin of the Transilvania University of Brasov: Series I - Engineering Sciences, and reviewer for prestigious ISI-rated journals (Advances in Mechanical Engineering, Applied Mathematical Modelling, Applied Mathematics and Computation, IEEE Transactions on Industrial Informatics, IEEE Transactions on Power Electronics, Journal of Mechanical Science and Technology, Journal of Renewable and Sustainable Energy, Mechanical Sciences, Mechanisms and Machine Theory, Robotics and Autonomous Systems, Solar Energy, Renewable Energy, and others). 